\title{
Evaluation of different chromogenic media for the detection of methicillin-resistant Staphylococcus aureus CC398 in broilers
}

\author{
L. J. Pletinckx • J. Dewulf • Y. De Bleecker • \\ G. Rasschaert • B. M. Goddeeris • I. De Man
}

Received: 24 September 2012 / Accepted: 13 February 2013 /Published online: 28 February 2013

(C) Springer-Verlag Berlin Heidelberg 2013

\begin{abstract}
Livestock-associated methicillin-resistant Staphylococcus aureus (LA-MRSA) has emerged in a wide variety of animal species, including poultry. The objective of this study was to evaluate three different chromogenic media for MRSA clonal complex (CC) 398 detection in broilers. On three Belgian poultry farms, 50 broiler chickens were sampled per farm from both nose shell and cloaca. All swab specimens were enriched and inoculated the following day on three chromogenic media: chromID MRSA (bioMérieux), Brilliance MRSA 2 Agar (Oxoid) and MRSASelect (Bio-Rad). ChromID had the highest isolation rates, yet, Brilliance MRSA 2 Agar demonstrated the highest relative sensitivity, while MRSASelect and Brilliance MRSA 2 Agar showed the highest relative specificity. A subset of MRSA isolates was confirmed to be CC398 by the polymerase chain reaction (PCR) targeting saul-hsdS1. In conclusion, Brilliance MRSA 2 Agar outperformed MRSASelect and chromID MRSA for the detection of MRSA in broilers.
\end{abstract}

L. J. Pletinckx $(\varangle) \cdot$ Y. De Bleecker $\cdot$ I. De Man

Department HIVB, Catholic University College

South-West-Flanders (KATHO), Wilgenstraat 32,

8800 Roeselare, Belgium

e-mail: larissa.pletinckx@katho.be

L. J. Pletinckx · B. M. Goddeeris

Department Biosystems, Division Gene Technology,

KU Leuven University, Kasteelpark Arenberg 30 - bus 2456,

3001 Heverlee, Belgium

\section{J. Dewulf}

Faculty of Veterinary Medicine, Veterinary Epidemiology Unit, Department of Reproduction, Obstetrics and Herd Health,

Ghent University, Salisburylaan 133,

9820 Merelbeke, Belgium

\section{G. Rasschaert}

Technology and Food Science Unit, Food Safety,

Institute for Agricultural and Fisheries Research (ILVO),

Brusselsesteenweg 370,

9090 Melle, Belgium

\section{Introduction}

Since 2005, an increasing number of reports on livestockassociated methicillin-resistant Staphylococcus aureus (LAMRSA) has been published, particularly in pigs, but also in cattle, horses and poultry. In Europe, the majority of LAMRSA belongs to clonal complex (CC) 398 [1]. The few studies that investigated the prevalence of LA-MRSA in poultry flocks appear to show a low within-flock prevalence [2-5]. This is in contrast to the high within-herd prevalence described in pigs in numerous studies [6-8]. The performances of chromogenic media for MRSA detection have previously been studied in pigs $[9,10]$ and veal calves [9]. It is of importance also to know which medium is optimal for use in broiler populations. Because, so far, no comparative studies have been performed in broilers, the present study aimed at evaluating three different chromogenic media [chromID MRSA (bioMérieux), Brilliance MRSA 2 Agar (Oxoid) and MRSASelect (Bio-Rad)] for MRSA detection in broilers.

\section{Materials and methods}

Sample collection and processing

This study was conducted between March and July 2011 on three mixed poultry-pig farms (Farms A, B and C) in Belgium. Samples were collected from 150 broiler chickens ( 50 per farm) between 5 and 6 weeks of age from two anatomical sampling sites (nose shell and cloaca) with separate swabs. The selection of animals was done as follows: in each corner and in the middle of the poultry stable, ten broiler chickens were sampled by convenience.

Sampling was performed by using a rayon-tipped sterile dry swab (Copan Innovation, Brescia, Italy) inserted into 
Mueller-Hinton broth (MHB; Oxoid, Basingstoke, UK) and supplemented with $6.5 \%$ sodium chloride. After sampling, the swabs were transported within the hour to the laboratory under cooled conditions. After overnight $\left(18-20 \mathrm{~h}\right.$ at $\left.37{ }^{\circ} \mathrm{C}\right)$ incubation of the swabs in the salt-enriched MHB, $1 \mu \mathrm{l}$ was inoculated the following day using the four-way-streakingplate-method on the three different chromogenic media (chromID MRSA, Brilliance MRSA 2 Agar and MRSASelect), following the instructions for use provided by the respective manufacturers. All media were allowed to warm to room temperature and were protected from light before inoculation. After $24 \mathrm{~h}$ of incubation at $37^{\circ} \mathrm{C}$, a total of 900 plates $(150$ broilers $\times 2$ sampling sites $\times 3$ different media) were read and interpreted, according to the manufacturers' instructions (technical files). Suspect colonies were identified by characteristic growth morphology and colour (pale to dark green and round colonies on chromID MRSA due to alpha-glucosidase production, denim-blue colonies due to phosphatase activity easy to read against the opaque background on Brilliance MRSA 2 Agar and small pink colonies on MRSASelect; the composition of the chromogenic mix is proprietary). The ATCC 43300 (MRSA) reference strain was included to verify the integrity of the media. One typical colony per suspected positive plate was purified for MRSA confirmation by plating on the corresponding MRSAselective medium, followed by plating on tryptone soy agar (TSA; CM0131; Oxoid, Basingstoke, UK) and overnight incubation at $37^{\circ} \mathrm{C}$.

\section{MRSA confirmation}

DNA was extracted from a phenotypically suspected colony according to the method of Strandén et al. [11] and DNA lysates were stored at $-20{ }^{\circ} \mathrm{C}$ until further use.

MRSA isolates were confirmed by multiplex polymerase chain reaction (PCR) targeting a Staphylococcus-specific 16S rRNA sequence, the mecA gene coding for methicillin resistance and the $S$. aureus-specific region of the thermonuclease (nuc) gene [12]. DNA amplification was performed using the Applied Biosystems StepOnePlus Real-Time PCR System.

To confirm the presence of MRSA CC398, a selection of 100 MRSA isolates were tested with a PCR targeting sau1$h s d S 1$, developed for the rapid detection of S. aureus CC398 by Stegger et al. [13]. This was an at-random selection of MRSA isolates evenly distributed over the different chromogenic media.

Data analysis and statistical analysis

When MRSA was isolated from an enriched swab specimen on at least one of the three media and confirmed by multiplex PCR, the swab was considered to be positive (gold standard). A true-positive result was defined as when an isolate picked from the given plate had a typical morphology and was confirmed to be MRSA by PCR. A falsepositive result was defined as when the isolate picked from the given plate had a typical morphology but proved not to be MRSA by PCR. A false-negative result was defined as a given plate without typical colonies and where no isolate was picked from but from the same sample MRSA was found on at least one other plate. Finally, a true-negative result was when no isolate was picked from the given plate and neither was MRSA detected on any of the other plates.

MRSA isolation rates (IR) per sampling site (nose shell, cloaca) and per chromogenic medium were calculated as the proportion of samples testing positive by phenotype (colony morphology and colour) on that sampling site and that medium, respectively.

For each sampling site and for each of the three media, the relative sensitivity, relative specificity and the $95 \%$ confidence intervals (CI) for MRSA detection were calculated. The relative sensitivity was calculated as the ratio of MRSA positives on that specific medium and sampling site (= true-positive results) to the total number of MRSA confirmed by PCR (gold standard) that were recovered from all media on the specific sampling site (= sum of the truepositive and the false-negative results). The relative specificity of a medium for a specific sampling site was calculated as the ratio of the true-negatives to the sum of the truenegative and the false-positive results. The $95 \% \mathrm{CI}$ of the relative sensitivity and relative specificity was also calculated $\left(\mathrm{x} \pm \frac{1.96 \sqrt{\mathrm{p}(1-\mathrm{p})}}{\mathrm{n}}\right)$ with $p$ being the sensitivity or specificity, respectively.

\section{Results}

IR and performance characteristics (number of MRSA truepositives, false-negatives and false-positives, relative sensitivity and relative specificity with corresponding $95 \% \mathrm{CI}$ ) per sampling site (nose shell, cloaca) and per chromogenic medium are shown in Table 1. In both sampling sites, the most MRSA-positive results by phenotype were found on chromID MRSA, yet, both the relative sensitivity and relative specificity of chromID MRSA were low compared with the other media. ChromID MRSA detected more falsepositives than Brilliance MRSA 2 Agar and MRSASelect. Furthermore, chromID MRSA and MRSASelect detected more false-negatives than Brilliance MRSA 2 Agar. Brilliance MRSA 2 Agar demonstrated, overall, the highest relative sensitivity for both sampling sites compared to MRSASelect and chromID MRSA. Regarding the relative specificity, MRSASelect and Brilliance MRSA 2 Agar showed the highest values for both sampling sites compared to chromID MRSA. 
Table 1 Isolation rates (IR) and performance characteristics [number of methicillin-resistant Staphylococcus aureus (MRSA) true-positives, false-negatives and false-positives, relative sensitivity and relative specificity with corresponding $95 \%$ confidence intervals (CI)] of three chromogenic MRSA screening media in a study carried out on three
Belgian poultry farms for all broiler swab specimens per anatomical sampling site (nose $n=150$ and cloaca $n=150$ ), including $n=50$ MRSA-positive samples for nose samples (gold standard) and $n=48$ for cloaca samples (gold standard)

\begin{tabular}{|c|c|c|c|c|c|c|c|}
\hline $\begin{array}{l}\text { Sampling } \\
\text { site }\end{array}$ & $\begin{array}{l}\text { Chromogenic } \\
\text { medium }\end{array}$ & $\begin{array}{l}\text { Isolation rates } \\
\text { (IR) }\end{array}$ & $\begin{array}{l}\text { Number of } \\
\text { MRSA true- } \\
\text { positives }\end{array}$ & $\begin{array}{l}\text { Number of MRSA } \\
\text { false-negatives }\end{array}$ & $\begin{array}{l}\text { Number of MRSA } \\
\text { false-positives }\end{array}$ & $\begin{array}{l}\text { Relative sensitivity, } \\
\%(95 \% \mathrm{CI})\end{array}$ & $\begin{array}{l}\text { Relative specificity, } \\
\%(95 \% \mathrm{CI})\end{array}$ \\
\hline \multirow[t]{3}{*}{ Nose } & $\begin{array}{l}\text { Brilliance } \\
\text { MRSA } 2\end{array}$ & $32.0 \%(48 / 150)$ & 47 & 3 & 1 & $94.0(83.5-98.7)$ & $99.0(94.6-100.0)$ \\
\hline & MRSASelect & $26.7 \%(40 / 150)$ & 40 & 10 & 0 & $80.0(66.3-90.0)$ & $100.0(96.4-100.0)$ \\
\hline & chromID MRSA & $40.7 \%(61 / 150)$ & 34 & 16 & 27 & $68.0(53.3-80.5)$ & $73.0(63.2-81.4)$ \\
\hline \multirow[t]{3}{*}{ Cloaca } & $\begin{array}{c}\text { Brilliance } \\
\text { MRSA } 2\end{array}$ & $32.0 \%(48 / 150)$ & 48 & 0 & 0 & $100.0(92.6-100.0)$ & $100.0(96.5-100.0)$ \\
\hline & MRSASelect & $24.7 \%(37 / 150)$ & 37 & 11 & 0 & $77.1(62.7-88.0)$ & $100.0(96.5-100.0)$ \\
\hline & chromID MRSA & $34.0 \%(51 / 150)$ & 39 & 9 & 12 & $81.3(67.4-91.1)$ & $88.2(80.4-93.8)$ \\
\hline
\end{tabular}

The PCR targeting saul-hsdS1 confirmed that all of the 100 tested MRSA isolates belonged to S. aureus CC398.

\section{Discussion}

The present study is the first comparing the performances of chromogenic media for MRSA detection in broilers. ChromID MRSA had the highest IR, while both the relative sensitivity and relative specificity of chromID MRSA were low in comparison to the other media. This high IR is explained by the fact that the definition of IR pertains to the phenotype, indicating that several false-positive isolates were found on this medium, which also explains the lower relative specificity. The lower relative sensitivity can be explained by the fact that chromID MRSA yielded the highest number of false-negative results. When taking the relative sensitivity and relative specificity into account, Brilliance MRSA 2 Agar outperformed MRSASelect and chromID MRSA for the detection of MRSA in broilers on both sampling sites. Thus, in terms of detecting the correct amount of MRSA positives, not only is a low number of false-positives (= high relative specificity) important, but also a low number of false-negatives (= high relative sensitivity) is necessary, as a low relative sensitivity will give an underestimation of the MRSA prevalence.

In a previous study of Graveland et al. [9], no statistically significant differences were found between the different media used for MRSA analysis of veal calf nasal swabs, while chromID MRSA had significantly lower rates of positive samples compared to MRSASelect and MRSA Screen (Oxoid, predecessor of Brilliance MRSA) for the analysis of pig nasal swabs. The current study cannot be compared to that of Graveland et al. [9], as another methodology for enrichment including antibiotics was used. In the study of Pletinckx et al. [10], the same methodology was used as in the present study, and chromID MRSA had the highest relative sensitivity and relative specificity for the detection of MRSA in pigs. On chromID MRSA, a rate of false-positives of only $1 \%$ (relative specificity=96\%) was found in pigs [10], in comparison to a false-positive rate of $13 \%(39 / 300)$ (relative specificity $=73$ and $88 \%$ ) in the current study. On MRSASelect (Bio-Rad), a false-positive rate of $10 \%$ (relative specificity $=64 \%$ ) was found in pigs [10], whereas in the present study, no false-positives (relative specificity $=100 \%$ ) were seen. Brilliance MRSA Agar used in the porcine study [10] can not, however, be compared to Brilliance MRSA 2 Agar in the present study, as Oxoid improved the latter medium by adding new inhibitory components and a new counter-stain, reducing the number of false-positives. Comparison of the performance characteristics of chromID MRSA and MRSASelect for pigs and broilers, respectively, indicates that the best MRSA screening method might be host-dependent, as differences in microbial flora could give rise to false-positives on the different media. Microbial flora might also differ according to the sampling site; however, in the current study, no difference was observed in the number of false-positives between nose and cloaca. The observer could represent a source of subjectivity, but all the plates in this study and in the porcine study [10] were read and interpreted by the same person, who was accustomed to working with chromogenic plates for the identification of MRSA. Differences in selective and elective components in the media may explain why some microorganisms give rise to false-positives on one medium and not on another.

The isolation of staphylococcal and non-staphylococcal organisms on chromogenic media designed for MRSA has been reported in a human MRSA study by Compernolle et al. [14]. Coagulase-negative staphylococci were a major cause of false-positive results, but, also, Enterobacter spp. resulted in false-positives on chromID MRSA [14]. In a 
former study in broilers [5], coagulase-negative staphylococci and enterococci were isolated from chromID MRSA (data not shown). Those species can harbour the mecA gene $[15,16]$, encoding beta-lactam resistance, which may partly explain how they could give rise to false-positives on chromID MRSA (which contains cefoxitin, a beta-lactam antibiotic). Beta-lactam resistance could, however, also be mediated by blaZ, typically carried by a plasmid [17]. This and other factors that could contribute to yielding falsepositives should be examined further. A high number of false-positives leads to extra costs, as more plates are used, and because all suspect MRSA isolates need to be confirmed by multiplex PCR.

\section{Conclusion}

The results show that screening with chromID MRSA, a chromogenic media that had the highest relative sensitivity and relative specificity in detecting livestock-associated methicillin-resistant Staphylococcus aureus (LA-MRSA) in pigs in a previous study [10], is less useful for LA-MRSA screening in poultry, due to a higher number of falsepositives and false-negatives. Brilliance MRSA 2 Agar outperformed MRSASelect and chromID MRSA for the detection of MRSA in broilers.

\begin{abstract}
Acknowledgements This research was funded by the Institute for the Promotion of Innovation by Science and Technology (Agentschap voor Innovatie door Wetenschap en Technologie, IWT) in Flanders (Belgium), project 070596. The authors wish to acknowledge Ine Vanherpe, Wim Vanhove and Laurent Lanckman for their help in the collection of the animal samples and technical assistance. Furthermore, we would also like to thank the farmers for their collaboration in this study.
\end{abstract}

Conflict of interest The authors declare that they have no conflict of interest and no financial or personal relations with people or organisations that could have inappropriately influenced this work.

\section{References}

1. Catry B, van Duijkeren E, Pomba MC, Greko C, Moreno MA, Pyörälä S, Ruzauskas M, Sanders P, Threlfall EJ, Ungemach F, Törneke K, Munoz-Madero C, Torren-Edo J; Scientific Advisory Group on Antimicrobials (SAGAM) (2010) Reflection paper on MRSA in food-producing and companion animals: epidemiology and control options for human and animal health. Epidemiol Infect 138:626-644

2. Nemati M, Hermans K, Lipinska U, Denis O, Deplano A, Struelens M, Devriese LA, Pasmans F, Haesebrouck F (2008) Antimicrobial resistance of old and recent Staphylococcus aureus isolates from poultry: first detection of livestock-associated methicillin-resistant strain ST398. Antimicrob Agents Chemother 52:3817-3819
3. Persoons D, Van Hoorebeke S, Hermans K, Butaye P, de Kruif A, Haesebrouck F, Dewulf J (2009) Methicillin-resistant Staphylococcus aureus in poultry. Emerg Infect Dis 15:452-453

4. Mulders MN, Haenen APJ, Geenen PL, Vesseur PC, Poldervaart ES, Bosch T, Huijsdens XW, Hengeveld PD, Dam-Deisz WDC, Graat EAM, Mevius D, Voss A, Van De Giessen AW (2010) Prevalence of livestock-associated MRSA in broiler flocks and risk factors for slaughterhouse personnel in The Netherlands. Epidemiol Infect 138:743-755

5. Pletinckx LJ, Verhegghe M, Dewulf J, Crombé F, De Bleecker Y, Rasschaert G, Goddeeris BM, De Man I (2011) Screening of poultry-pig farms for methicillin-resistant Staphylococcus aureus: sampling methodology and within herd prevalence in broiler flocks and pigs. Infect Genet Evol 11(8):2133-2137

6. Voss A, Loeffen F, Bakker J, Klaassen C, Wulf M (2005) Methicillin-resistant Staphylococcus aureus in pig farming. Emerg Infect Dis 11:1965-1966

7. de Neeling AJ, van den Broek MJM, Spalburg EC, van SantenVerheuvel MG, Dam-Deisz WDC, Boshuizen HC, van de Giessen AWV, van Duijkeren E, Huijsdens XW (2007) High prevalence of methicillin resistant Staphylococcus aureus in pigs. Vet Microbiol 122:366-372

8. Köck R, Harlizius J, Bressan N, Laerberg R, Wieler LH, Witte W, Deurenberg RH, Voss A, Becker K, Friedrich AW (2009) Prevalence and molecular characteristics of methicillin-resistant Staphylococcus aureus (MRSA) among pigs on German farms and import of livestock-related MRSA into hospitals. Eur J Clin Microbiol Infect Dis 28(11):1375-1382

9. Graveland H, van Duijkeren E, van Nes A, Schoormans A, Broekhuizen-Stins M, Oosting-van Schothorst I, Heederik D, Wagenaar JA (2009) Evaluation of isolation procedures and chromogenic agar media for the detection of MRSA in nasal swabs from pigs and veal calves. Vet Microbiol 139:121-125

10. Pletinckx LJ, De Bleecker Y, Dewulf J, Rasschaert G, Goddeeris BM, De Man I (2012) Evaluation of salt concentrations, chromogenic media and anatomical sampling sites for detection of methicillinresistant Staphylococcus aureus in pigs. Vet Microbiol 154:363-368

11. Strandén A, Frei R, Widmer AF (2003) Molecular typing of methicillin-resistant Staphylococcus aureus: can PCR replace pulsed-field gel electrophoresis? J Clin Microbiol 41:3181-3186

12. Maes N, Magdalena J, Rottiers S, De Gheldre Y, Struelens MJ (2002) Evaluation of a triplex PCR assay to discriminate Staphylococcus aureus from coagulase-negative staphylococci and determine methicillin resistance from blood cultures. J Clin Microbiol 40:1514-1517

13. Stegger M, Lindsay JA, Moodley A, Skov R, Broens EM, Guardabassi L (2010) Rapid PCR detection of Staphylococcus aureus clonal complex 398 by targeting the restriction-modification system carrying saul-hsdS1. J Clin Microbiol 49(2):732-734

14. Compernolle V, Verschraegen G, Claeys G (2007) Combined use of Pastorex Staph-Plus and either of two new chromogenic agars, MRSA ID and CHROMagar MRSA, for detection of methicillinresistant Staphylococcus aureus. J Clin Microbiol 45:154-158

15. Hanssen AM, Kjeldsen G, Sollid JU (2004) Local variants of staphylococcal cassette chromosome mec in sporadic methicillin-resistant Staphylococcus aureus and methicillin-resistant coagulase-negative staphylococci: evidence of horizontal gene transfer? Antimicrob Agents Chemother 48:285-296

16. Kassem II, Esseili MA, Sigler V (2008) Occurrence of mecA in nonstaphylococcal pathogens in surface waters. J Clin Microbiol 46(11):3868-3869

17. Katayama Y, Zhang HZ, Hong D, Chambers HF (2003) Jumping the barrier to beta-lactam resistance in Staphylococcus aureus. J Bacteriol 185:5465-5472 